\title{
A REPRESENTAÇÃO DA CONSULTA DE ENFERMAGEM PARA OS IDOSOS DO HIPERDIA
}

\section{THE REPRESENTATION OF THE NURSING CONSULTATION FOR THE ELDERLY OF HIPERDIA}

\section{LA REPRESENTACIÓN DE LA CONSULTA DE LA PACIENTE PARA LAS PERSONAS MAYORES}

\author{
Camilo Eduardo Almeida Pereira ${ }^{1}$, Aryanne Magno Santos Silva ${ }^{2}$, Diego Carvalho de Sousa ${ }^{3}$, \\ Mayara Melo Galvão ${ }^{4}$
}

Fonte de finaciamento: Finaciamento próprio

Manuscrito proveniente da monografía

\begin{abstract}
RESUMO
Objetivo: descrever a representação da consulta de enfermagem para os idosos acompanhados pelo programa do Hiperdia de uma Unidade de saúde da Amazônia. Metodologia: Trata-se de um estudo descritivo de abordagem qualitativa, desenvolvido em uma UBS de AbaetetubaPará, ocorreu entre setembro e novembro de 2018, os dados foram analisados pelo método de análise de conteúdo. Resultado: Emergiram três categorias, a primeira sobre o papel do enfermeiro no programa Hiperdia na ótica do idoso, as palavras mais citadas são: avaliar, cuidar, conversar, orientar, saúde e alimentação. A segunda é sobre a representatividade da consulta de enfermagem, nessa predominou as palavras: cuidado, saúde, mudança, essencial e conversa. A última relata a contribuição do enfermeiro para a mudança do estilo de vida do idoso, tendo como destaque as seguintes palavras: qualidade de vida, alimentação, caminhada, remédio, exercício. Conclusão: A consulta de enfermagem mostrou-se fundamental, ainda necessita de aperfeiçoamentos, contudo, as ações executadas já demonstram resultados expressivos na mudança de estilo de vida dos idosos.
\end{abstract}

Descritores: Enfermagem; Idosos; Hipertensão; Diabetes Mellitus

\begin{abstract}
Objective: This study aims to describe the representation of the nursing consultation for the elderly accompanied by the Hiperdia program of an Amazon Health Unit. Methodology: It is a descriptive study of a qualitative approach, developed in a basic health unit of Abaetetuba-Pará, occurred between September and November of 2018, the data were analyzed by the content analysis method. Results: There were three categories, the first on the role of the nurse in the Hiperdia program in the elderly, the most cited words are: evaluate,

\footnotetext{
${ }^{1}$ Mestre em saúde Coletiva - Universidade Federal do Pará. Especialista em enfermagem do trabalho - Faculdade Integrada do Rio de Janeiro. Bacharel em Enfermagem - Escola de Enfermagem Magalhães Barata da Universidade do Estado do Pará. Enfermeiro. Professor adjunto da Faculdade Pan Amazônica e da Faculdade de Medicina da Universidade Federal do Pará.

${ }^{2}$ Bacharelado em Enfermagem. Faculdade Pan Amazônica.

${ }^{3}$ Graduando em Enfermagem. Faculdade Pan Amazônica, FAPAN, Brasil.

${ }^{4}$ Especialização em Residência Multiprofissional em Atenção a Saúde Cardiovascular pela Fundação Pública Estadual Hospital de Clínicas Gaspar Vianna, FHCGV, Brasil. Graduação em Escola de Enfermagem 'Magalhães Barata'. Universidade do Estado do Pará, UEPA, Brasil.
} 
care, talk, guide, health and food. The second is about the representativeness of the nursing consultation, in that predominated the words: care, health, change, essential and conversation. The last one reports the contribution of the nurse to the lifestyle change of the elderly, highlighting the following words: quality of life, food, walking, medicine, exercise.Conclusion: The nursing consultation was fundamental, it still needs improvement, however, the actions performed already demonstrate significant results in the change of lifestyle of the elderly.

Descriptors: Nursing; Seniors; Hypertension; Diabetes Mellitus.

\section{RESUMEN}

Objetivo: Este estudio se objetiva describir la representación de la consulta de enfermería para los ancianos acompañados por el programa del Hiperdia de una Unidad de salud Amazonia. Metodología: Es un estudio descriptivo de un enfoque cualitativo, desarrollado en una unidad de salud básica de Abaetetuba-Pará, ocurrido entre septiembre y noviembre de 2018, los datos se analizaron mediante el método de análisis de contenido. Resultados: En el caso de la enfermería en el programa Hiperdia en la óptica del anciano, las palabras más citadas son: evaluar, cuidar, conversar, orientar, salud y alimentación. La segunda es sobre la representatividad de la consulta de enfermería, en la que predominó las palabras: cuidado, salud, cambio, esencial y conversación. La última relata la contribución del enfermero para el cambio del estilo de vida del anciano, teniendo como destaque las siguientes palabras: calidad de vida, alimentación, caminata, remedio, ejercicio. Conclusión: La consulta de enfermería se mostró fundamental, aún necesita de mejoras, sin embargo, las acciones ejecutadas ya demuestran resultados expresivos en el cambio de estilo de vida de los ancianos.

Descriptores: Enfermería; los ancianos; Hipertensión; Diabetes Mellitus.

\section{INTRODUÇÃO}

A Diabetes mellitus (DM) e a Hipertensão Arterial Sistêmica (HAS) são responsáveis pela primeira causa de mortalidade e de hospitalizações no Sistema Único de Saúde (SUS), e representam mais da metade do diagnóstico primário em pessoas com insuficiência renais crônicas submetidas à diálise, além de provocarem outras complicações como amputações de membros inferiores, Doença Arterial Coronária (DAC), na Insuficiência Cardíaca (IC), na Retinopatia Hipertensiva e na Insuficiência Vascular Periférica. ${ }^{1}$

Sendo que as doenças cardiovasculares são a principal causa de óbito no Brasil e no mundo, tendo o DM e a HAS como importantes fatores de risco, assim é um desafio para o sistema público de saúde brasileiro garantir o acompanhamento sistemático dos indivíduos identificados como portadores desses agravos, assim como o desenvolvimento de ações referentes à promoção da saúde e à prevenção de Doenças Crônicas Não Transmissíveis (DCNT). ${ }^{2}$

Tendo em vista, a importância de um acompanhamento constante, e levando em consideração o auto índice de agravos em usuários portadores de doenças cardiovasculares, em 2002 foi criado o 
Plano de Reorganização da Atenção à HAS

e DM, denominado Hiperdia. Este programa objetiva diminuir os agravos, estabelecendo metas e diretrizes para ampliar ações de prevenção, diagnóstico, tratamento e controle dessas patologias, através da reorganização do trabalho de atenção à saúde, das unidades da rede básica dos Serviços de Saúde. ${ }^{3}$

O Hiperdia constitui-se em um programa de cadastramento e acompanhamento de hipertensos e/ou diabéticos que visa o controle da DM e HAS e uma melhor qualidade de vida aos usuários. Através da vinculação do usuário à Unidade Básica de Saúde (UBS) e à Estratégia de Saúde da Família (ESF) que ele está inserido na perspectiva de assim poder realizar uma assistência continuada e com qualidade, fornecendo medicamentos, acompanhamento e fazer avaliação de risco entre os usuários cadastrados. ${ }^{4}$

Nesta perspectiva, a equipe de Saúde da Família possui papel fundamental no desenvolvimento das ações de prevenção e controle de agravos. Para tanto, cabe-lhe sistematizar a assistência e organizar o atendimento, de modo que o usuário hipertenso e/ou diabético tenha acesso a todos os serviços, como recebimento de medicamentos anti-hipertensivos e/ou antidiabéticos, mensuração de peso, altura, circunferência abdominal, pressão arterial e glicemia capilar e outros, bem como a disponibilidades de consultas médicas e de enfermagem. ${ }^{5}$

A Consulta de Enfermagem surge como uma estratégia tecnológica de cuidado importante e resolutiva, objetivando uma assistência sistematizada de enfermagem, de forma global e individualizada, respaldada por lei, privativa do enfermeiro, e que oferece inúmeras vantagens na assistência prestada, facilitando a promoção da saúde, o diagnóstico e o tratamento precoce, além da prevenção de situações evitáveis. ${ }^{6}$

A atuação do enfermeiro na consulta de enfermagem não pode estar dissociada da educação em saúde, pois essa estratégia se insere no contexto da atuação da enfermagem, permitindo uma aproximação com o usuário, fazendo com que haja uma relação dialógica-reflexiva sobre o processo de saúde-doença das DCNTs, para que assim o usuário perceba-se como sujeito de transformação de sua própria vida, ou seja, a consulta de enfermagem precisa ser centrada no idoso, para que o mesmo possa ser protagonista do seu plano terapêutico. ${ }^{7}$

No entanto, apesar da importância da consulta de enfermagem no programa do Hiperdia, nem sempre conseguimos mensurar o impacto de tal consulta na mudança de estilo de vida dos idosos. Desta forma, à avaliação é importante para que haja um planejamento das ações dos profissionais, pautadas nas necessidades do 
usuário, família e comunidade. Assim, esse estudo tem como objetivo descrever a representação da consulta de enfermagem para os idosos acompanhados pelo programa do Hiperdia de uma unidade de saúde da Amazônia, Pará.

\section{MATERIAL E METODO}

Trata-se de um estudo descritivo de abordagem qualitativa, desenvolvido em uma UBS de Abaetetuba-Pará, localizada no nordeste do Pará, a pesquisa ocorreu entre setembro e novembro de 2018. Participaram deste estudo 17 usuários com HAS e DM cadastrados no Programa Hiperdia da UBS, sendo que o número total de usuários cadastrados é de 50.

Para seleção da amostra utilizou-se como critério de inclusão, possuir cadastro ativo na UBS, ter uma idade maior ou igual a 65 anos, ter capacidade cognitiva preservada para compreender as perguntas e responde-las de forma adequada, além de três consultas regulares com o enfermeiro, conforme preconizado pelo Hiperdia. Foram excluídos dessa seleção, os usuários que recebem visitas domiciliares.

A coleta de dados ocorreu mediante aproximação com o usuário do programa Hiperdia. Primeiramente foi explicada a importância do estudo, assim como a relevância da participação dos mesmos. Após o primeiro contato com usuário foi realizado um agendamento prévio, visando o melhor dia e horário, para proceder à entrevista.

A entrevista foi semiestruturada com roteiro prévio, dividido em duas partes. A primeira com dados dos usuários a serem entrevistados (sexo, idade, estado civil, nível de escolaridade, DCNT, tempo de acompanhamento na UBS e frequência de consulta com o enfermeiro). Já a segunda parte composta de cinco perguntas especificas que foram: Você poderia relatar o que é realizado na consulta de enfermagem? $O$ que a consulta de enfermagem representa na sua via? Qual a sua opinião sobre o atendimento da consulta de enfermagem? Como a consulta de enfermagem contribui para a sua mudança de estilo de vida? Como você acha que poderia ser melhorada a consulta de enfermagem?

Ressalta-se que as entrevistas foram gravadas e posteriormente realizada as transcrições. Análise de dados foi de acordo com a proposta da Bardin, que tem como pressuposto analise de conteúdo. ${ }^{9}$ A exploração dos discursos foi codificada, a fim de alcançar o núcleo de compreensão, organizados em nuvens de palavras, utilizando o programa "wordclouds" e, agregados em eixos temáticos, sendo que as palavras que receberam mais destaque são representadas por uma fonte maior, permitindo uma descrição das características pertinentes do conteúdo. 
O estudo foi previamente autorizado e aprovado pelo Comitê de Ética em Pesquisa da Universidade Paulista (UNIP) (Parecer $\mathrm{n}^{\mathrm{o}}: 2.895 .953$, CAAE: 97705418.6.0000.5512). Esse estudo respeitou os princípios éticos de acordo com resolução 466/12 (CNS/MS), desta maneira o estudo correu mediante assinatura dos participantes do Termo Livre e Esclarecido, e a fim de preservar a identidade dos participantes utilizou-se o código alfanumérico com a letra $\mathrm{H}$ de Hiperdia e o número sequencial das entrevistas (Ex: H1, $\mathrm{H} 2, \mathrm{H} 3)$.

\section{RESULTADO}

Dos 17 entrevistados cadastrados no programa Hiperdia, $14(82,35 \%)$ são do sexo feminino e $3(17,65 \%)$ são do sexo masculino. Em relação a faixa etária 12 pessoas possuíam entre 65-70 anos (70,58\%), 1 pessoa entre 71-75 anos $(5,88 \%)$ e 4 pessoas entre $76-80(23,52)$. Quanto ao estado civil, $4(23,53 \%)$ são solteiros, $11(64,71 \%)$ são casados e 1 $(5,88 \%)$ divorciado.

A renda mensal dos participantes em sua maioria, é de acima de um salário mínimo em um total de $11(64,71 \%)$ e 6 $(35,29 \%)$ abaixo de um salário mínimo. No que se refere ao nível de escolaridade dos participantes, $15(88,24 \%)$ possuem nível fundamental e $2(11,76 \%)$ possuem nível médio. Ressaltamos que esse tópico é para caracterizar o público alvo da pesquisa, conforme observa-se no Quadro 1.

\section{Quadro 1: Caracterização dos Participantes}

\begin{tabular}{|c|c|c|c|c|c|}
\hline Participante & Sexo & Idade & Estado Civil & Renda & Escolaridade \\
\hline $\mathrm{H} 1$ & $\mathrm{~F}$ & 77 & $\mathrm{~S}$ & ACSM & $\mathrm{F}$ \\
\hline $\mathrm{H} 2$ & $\mathrm{~F}$ & 68 & $\mathrm{C}$ & ABSM & $\mathrm{F}$ \\
\hline H3 & $\mathrm{M}$ & 65 & $\mathrm{C}$ & ACSM & $\mathrm{F}$ \\
\hline H4 & M & 80 & $\mathrm{C}$ & ACSM & $\mathrm{F}$ \\
\hline H5 & $\mathrm{F}$ & 78 & $\mathrm{C}$ & ACSM & $\mathrm{F}$ \\
\hline H6 & $\mathrm{F}$ & 65 & $\mathrm{C}$ & ACSM & $\mathrm{F}$ \\
\hline H7 & $\mathrm{F}$ & 69 & $\mathrm{C}$ & ACSM & $\mathrm{F}$ \\
\hline H8 & $\mathrm{F}$ & 69 & $\mathrm{C}$ & ACSM & $\mathrm{F}$ \\
\hline H9 & $\mathrm{F}$ & 68 & $\mathrm{C}$ & ABSM & $\mathrm{F}$ \\
\hline H10 & $\mathrm{F}$ & 73 & D & ABSM & M \\
\hline H11 & $\mathrm{F}$ & 65 & $\mathrm{~S}$ & ABSM & $\mathrm{F}$ \\
\hline H12 & $\mathrm{F}$ & 79 & S & ACSM & $\mathrm{F}$ \\
\hline H13 & $\mathrm{F}$ & 65 & $\mathrm{~S}$ & ABSM & $\mathrm{M}$ \\
\hline H14 & M & 66 & $\mathrm{C}$ & ACSM & $\mathrm{F}$ \\
\hline $\mathrm{H} 15$ & $\mathrm{~F}$ & 66 & $\mathrm{C}$ & ACSM & $\mathrm{F}$ \\
\hline H16 & $\mathrm{F}$ & 69 & $\mathrm{C}$ & ACSM & $\mathrm{F}$ \\
\hline H17 & $\mathrm{F}$ & 65 & $\mathrm{C}$ & ABSM & $\mathrm{F}$ \\
\hline \multicolumn{6}{|c|}{$\begin{array}{l}\text { SEXO: } \mathrm{F}=\text { feminino } \mathrm{M}=\text { masculino; ESTADO CÍVIL: } \mathrm{S}=\text { solteiro } \mathrm{C}=\text { casado } \mathrm{D}=\text { =divorciado; RENDA: } \\
\text { ACSM=acima de um salário mínimo ABSM=abaixo de um salário mínimo; ESCOLARIDADE: } \\
\text { F=fundamental } \mathrm{M}=\text { =médio } \mathrm{S}=\text { superior }\end{array}$} \\
\hline
\end{tabular}

Fonte: Elaborado pelos autores 


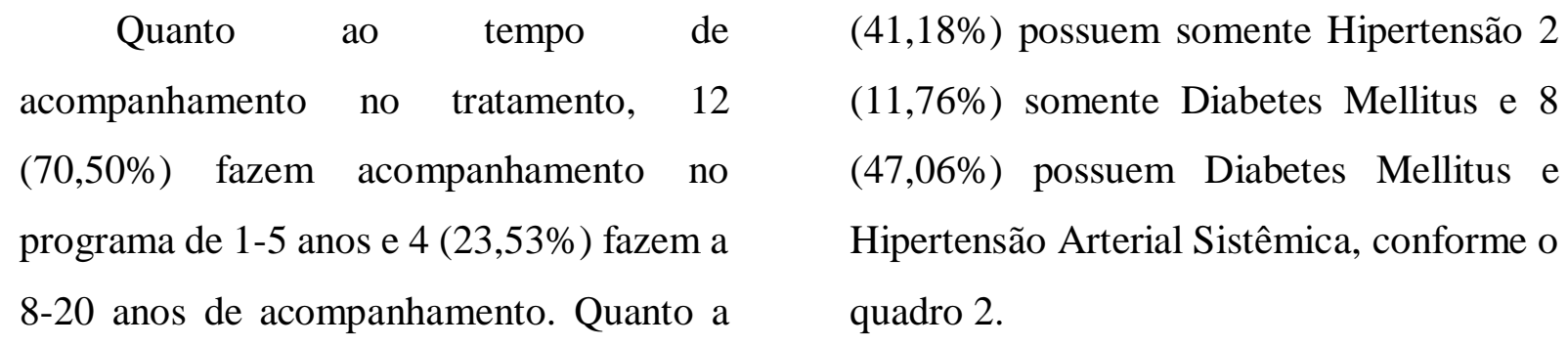

Doença crônica não transmissível, 7

\section{Quadro 2: Caracterização quanto a doença crônica não transmissível}

\begin{tabular}{|c|c|c|}
\hline Participante & Tempo de Acompanhamento (anos) & $\begin{array}{c}\text { Doença Crônica não } \\
\text { transmissível }\end{array}$ \\
\hline H1 & 3 & HAS e DM \\
\hline H2 & 3 & HAS e DM \\
\hline H3 & 3 & DM \\
\hline H4 & 1 & HAS e DM \\
\hline H5 & 4 & HAS e DM \\
\hline H6 & 20 & HAS e DM \\
\hline H7 & 8 & HAS e DM \\
\hline H8 & 10 & HAS e DM \\
\hline H9 & 3 & HAS \\
\hline H10 & 3 & HAS \\
\hline H11 & 3 & HAS e DM \\
\hline H12 & 20 & HAS \\
\hline H13 & 5 & DM \\
\hline H14 & 4 & HAS \\
\hline H15 & 4 & HAS \\
\hline H16 & 3 & HAS \\
\hline H17 & 5 & HAS \\
\hline
\end{tabular}

Fonte: Elaborado pelos autores, 2018

Diante desse contexto emergiram as

Nesta primeira categoria destacaseguintes categorias temáticas: $\mathrm{O}$ papel do se o papel do enfermeiro na perspectiva do enfermeiro no programa Hiperdia na ótica idoso do programa Hiperdia, podendo ser do idoso; A representatividade da consulta e enfermagem para o idoso do programa Hiperdia; A contribuição do enfermeiro para a mudança do estilo de vida do idoso do programa Hiperdia.

Categoria 1:0 papel do enfermeiro no programa Hiperdia na ótica do idoso verificado no quadro 3, demonstrando que os idosos têm uma visão do enfermeiro de um profissional que avalia, cuida, pergunta, conversa, orienta sobre o processo saúdedoença, tem uma preocupação com o estilo de vida. 
Quadro 3. O papel do enfermeiro no programa Hiperdia na ótica do idoso da Unidade de Saúde de Abaetetuba-Pará , 2018.

\begin{tabular}{|c|c|c|}
\hline Participante & Depoimento & Nuvem de palavras \\
\hline $\mathrm{H} 2$ & $\begin{array}{c}\text { "Eu chego aqui e a doutora } \\
\text { enfermeira me pergunta sobre o } \\
\text { que ando comendo, se ando } \\
\text { cuidando de mim" }\end{array}$ & \\
\hline $\mathrm{H} 7$ & $\begin{array}{c}\text { "Ela cuida da gente aqui } \\
\text { entende? se preocupa, faz um } \\
\text { monte de pergunta, e me ajuda a } \\
\text { me cuidar também, dizendo o que } \\
\text { eu tenho que fazer" }\end{array}$ & nemédios. \\
\hline H9 & $\begin{array}{l}\text { A enfermeira me pergunta como } \\
\text { eu estou me sentindo, se eu tomei } \\
\text { meus remédios, se eu senti } \\
\text { alguma coisa diferente nesses } \\
\text { dias, diferente do normal no } \\
\text { caso" }\end{array}$ & $\begin{array}{c}\text { assistidassallace } \\
\text { penguntam } \\
\text { periguntas } \\
\text { alimentação connige }\end{array}$ \\
\hline H11 & $\begin{array}{l}\text { Bom, ela pega os meus dados, } \\
\text { avalia e me pergunta como eu } \\
\quad \text { estou me sentindo" }\end{array}$ & \\
\hline H12 & $\begin{array}{l}\text { "ela faz umas perguntas sobre a } \\
\text { minha vida, pergunta se eu estou } \\
\text { cuidando da minha saúde, e se eu } \\
\text { não tiver ela me corrige" }\end{array}$ & \\
\hline
\end{tabular}

Fonte: Elaborado pelos autores, 2018.

Categoria 2: A representatividade da consulta e enfermagem para $o$ idoso do programa Hiperdia

Em relação à representatividade da consulta de enfermagem para o idoso do programa Hiperdia, relataram que o enfermeiro é um profissional confiável, que conversa, que se preocupa, ou seja, é um profissional que cuida, podendo ser observado no quadro 4.

Quadro 4: A representatividade da consulta e enfermagem para o idoso do programa Hiperdia, Abaetetuba-Pará, 2018

\begin{tabular}{|c|c|c|}
\hline Participante & Depoimento & \\
\hline $\mathrm{H} 2$ & $\begin{array}{c}\text { "Representa a mudança que eu } \\
\text { precisava" }\end{array}$ & \\
\hline $\mathrm{H} 7$ & $\begin{array}{c}\text { "É essencial, porque me ajuda a } \\
\text { ter os devidos cuidados" }\end{array}$ & \\
\hline
\end{tabular}




\begin{tabular}{|c|c|c|}
\hline $\mathrm{H} 9$ & $\begin{array}{c}\text { "E importante porque me ajuda } \\
\text { a manter minha saúde } \\
\text { regulada" }\end{array}$ \\
\hline $\mathrm{H} 11$ & $\begin{array}{c}\text { "O que ela representa, } \\
\text { representa que ela cuida de mim } \\
\text { né!" }\end{array}$ \\
\hline $\mathrm{H} 12$ & $\begin{array}{c}\text { "Eu acho maravilhoso, ela tira } \\
\text { minhas duvidas me orienta } \\
\text { sobre como tenho que agir em } \\
\text { relação a minha saúde" }\end{array}$ \\
\hline $\mathrm{H} 15$ & $\begin{array}{c}\text { "Representa basicamente o } \\
\text { cuidado, sou bem cuidada aqui, } \\
\text { em casa não tenho esse cuidado } \\
\text { sabe?". }\end{array}$ \\
\hline $\mathrm{H} 1$ & $\begin{array}{c}\text { "É muito importante na minha } \\
\text { vida, me ajuda a manter minha } \\
\text { saúde". }\end{array}$ \\
\hline $\mathrm{H} 17$ & $\begin{array}{c}\text { "E muito boa porque a gente } \\
\text { tem com quem conversar, eles se } \\
\text { preocupam com a gente. }\end{array}$ \\
\hline
\end{tabular}

Fonte: Elaborada pelos autores, 2018.

\section{Categoria 3: A contribuição do} enfermeiro para a mudança do estilo de vida do idoso do programa Hiperdia

Pode-se aferir que o enfermeiro é o profissional motivador para mudança do estilo de vida dos idosos, já que estes relataram que o enfermeiro contribuiu para mudança na alimentação, melhora na qualidade de vida, hábitos saudáveis como exercícios físicos, o cuidado com a medicação, como está descrito na nuvem de palavra da imagem do quadro 5.

Quadro 5: A contribuição do enfermeiro para a mudança do estilo de vida do idoso do programa Hiperdia da Unidade de Saúde de Abaetetuba-Pará, 2018

\begin{tabular}{|c|c|c|}
\hline Participante & Depoimento & Nuvem de palavras \\
\hline $\mathrm{H} 2$ & $\begin{array}{c}\text { "Ela é boa assim, depois que } \\
\text { eu comecei a me consultar } \\
\text { com a enfermeira, eu nunca } \\
\text { mais esqueci de tomar meu } \\
\text { remédio, me alimento } \\
\text { melhor. }\end{array}$ & \\
\hline $\mathrm{H} 7$ & $\begin{array}{c}\text { "sim eu mudei minha } \\
\text { alimentação, me exercito, me } \\
\text { cuido" }\end{array}$ \\
\hline $\mathrm{H} 9$ & $\begin{array}{c}\text { "Contribuiu sim, eu passei a } \\
\text { me cuidar melhor". }\end{array}$ & \\
\hline
\end{tabular}




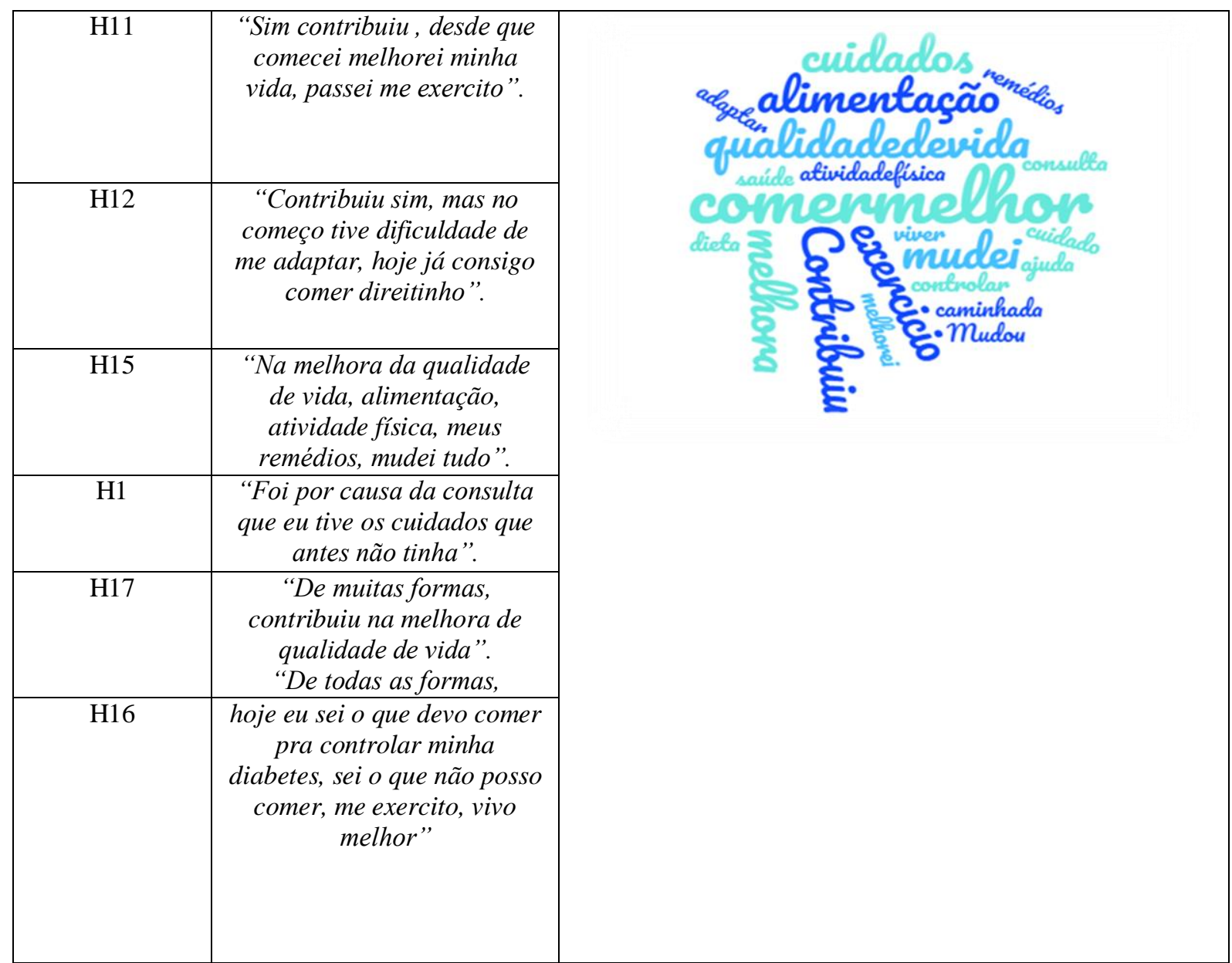

Fonte: elaborado pelos autores, 2018

\section{DISCUSSÃO}

A consulta de enfermagem tem o objetivo de prestar uma assistência sistematizada e individualizada, identificando problemas de saúde-doença, através da entrevista para anamnese, o exame físico, estabelecimento processo de enfermagem, que consiste nas etapas que são: investigação, diagnóstico, planejamento, implementação e avaliação. ${ }^{10}$ Desta maneira, o enfermeiro que adota essas etapas terá mais resolutividade no plano de cuidado do idoso. Nessa perspectiva pode-se averiguar a importância da assistência do enfermeiro no acompanhamento do idoso do programa Hiperdia, pois aproximação entre usuário e profissional proporciona uma observação detalhada e contínua, que possibilita a prevenção e identificação de possíveis agravos e complicações.

$\mathrm{Na}$ nuvem de palavras exposta na categoria 1, a palavra "orientação" recebeu destaque. Os idosos relatam que o enfermeiro do programa o orienta, quanto ao cuidado com a saúde, alimentação, medicação, estilo de vida, ou seja, são orientações para o autocuidado, visando que o usuário seja protagonista do plano terapêutico. 
A teoria de Orem, conhecida por teoria de autocuidado, apresenta como conceito básico a prática de atividades executadas pelo próprio indivíduo em seu benefício, para a manutenção da vida, da saúde e do bem-estar. Está atrelado aos fatores que interferem na capacidade de desempenhá-lo, entre os quais se destaca a idade, as experiências de vida, a cultura, o gênero, o padrão de vida, a educação e a crença dos seres humanos. ${ }^{11}$

O cuidar do usuário com DCNT, deve ter como princípio básico assistir o idoso, família e comunidade, auxiliá-los no desenvolvimento de habilidades e atitudes que proporcionem um autocuidado efetivo deste problema crônico de saúde. A comunidade na qual esse idoso está inserido deve ser envolvida no processo, de forma que as ações ultrapassem o tratamento da doença, visando a prevenção e promoção a saúde, com intuito de diminuir a prevalência das DCNTs.

Nesse contexto, a atuação do enfermeiro na educação em saúde possibilita que o idoso tenha uma visão ampla quanto ao seu tratamento e consiga refletir em relação aos seus hábitos e estilo de vida frente à HAS e a DM, e suas consequências a longo prazo, fazendo com que esse idosos sejam multiplicadores de informação aos seus pares, perpetuando a cadeia de promoção e prevenção a saúde dentro da sua comunidade.
No entanto, ressalta-se que o estímulo ao autocuidado é complexo e trabalhoso, tanto para o profissional de saúde como para os usuários do programa Hiperdia. É difícil alterar estilos de vida e manter essas modificações, sendo de suma importância que os profissionais promovam estratégicas educacionais individuais ou coletivas para que o idoso possa efetivamente seguir o plano de cuidado.

Autores afirmam que o enfermeiro da UBS tem como desafio programar o cuidado na construção de relações interpessoais de diálogo, fazendo uma escuta humanizada e respeitosa. $\mathrm{O}$ processo de cuidar é a forma como se dá o cuidado, de forma que haja uma interação entre o cuidador e o ser que recebe o cuidado. ${ }^{12}$

O primeiro tem um papel ativo, pois desenvolve ações acompanhadas de comportamentos de cuidar. Já o segundo, o ser cuidado, tem um papel mais passivo, em função de sua situação, pode tornar-se dependente, temporariamente, mudando para um papel menos passivo, contribuindo no cuidado, e ser responsável pelo próprio cuidado. $^{13}$

Nesta perspectiva, podemos notar que o cuidar está presente na prática de enfermagem, uma vez que a maioria dos participantes descreve o enfermeiro como um cuidador. O cuidado de enfermagem favorece a formação de vínculo do idoso do programa Hiperdia com o enfermeiro, o que 
decorre tanto do convívio com o usuário, quanto com a comunidade, como das ações e estratégias desenvolvidas pelo profissional.

A população precisa sentir-se confiante e segura quanto ao trabalho desenvolvido pelos profissionais de saúde, pois o oposto pode acarretar sofrimento ou até mesmo depressão. Assim, devido à sua proximidade singular durante o cuidado prestado, os enfermeiros desempenham um papel importantíssimo na percepção desses sentimentos de seus clientes e na efetiva formação do vínculo para a manutenção da saúde. ${ }^{14}$

A saúde é algo dinâmico, indivisível, que existe quando são mantidas as condições do meio ambiente e o acesso às ações que garantem o estado de saúde. Entre as teorias da enfermagem, existe uma certa unanimidade conceitual quanto ao desempenho da profissão. Em princípio, ela assume a responsabilidade de se solidarizar com as pessoas, grupos, famílias e comunidades, com o objetivo de mobilizar a cooperação de cada ser humano para conquistar e conservar o estado de saúde. ${ }^{15}$

Assim, é preciso que o enfermeiro possa fazer um diálogo entre os determinantes sociais em saúde, para que possa desenvolver ações pautadas nas condições dos idosos, valorizando os saberes culturais que o mesmo tem acerca da sua doença, interagindo com o senso comum e cientifico, para que possa desenvolver o plano de cuidado, na qual a tomada de decisão seja em conjunto para que possa promover a saúde do idoso.

O Hiperdia tem como um dos objetivos proporcionar a qualidade de vida dos idosos, pois nesse programa são vivenciadas ações de promoção da saúde que instigam práticas de atividades físicas, alimentação e hábitos saudáveis. Essas práticas interferem de forma positiva nas condições de saúde do usuário. ${ }^{16}$

A prática de exercício físico e hábitos alimentares saudáveis devem ser percebidos como terapêuticas não farmacológicas fundamentais no tratamento do idoso do Hiperdia. As atividades físicas representam relevância tanto na redução da pressão arterial quanto no controle glicêmico, além do controle dos fatores de risco de seus praticantes ${ }^{17}$.Dessa forma, as ações de enfermagem voltadas aos portadores de HAS e DM, devem incluir estratégias de promoção de estilos de vida saudáveis e de encorajamento.

O estilo de vida é compreendido como um modo de viver que conduz à maneira de ser do sujeito, aos hábitos e suas expressões. A forma de vida da pessoa varia de acordo com o grupo social e cultural em que a mesma está inserida. A decisão do indivíduo para manter uma forma peculiar de vida envolve tanto aspectos externos como mentais. 
Quando há um desequilibro do indivíduo com meio em que vive, pode ocorrer formas adaptativas diante das tensões do cotidiano, fazendo com que haja uma ligação com fatores de riscos que agrava o quadro clinico dos idosos com HAS e DM. Sabe-se que esses fatores estão associados principalmente ao tabagismo, etilismo, alimentação inadequada, sedentarismo e estresse. ${ }^{18}$

Logo, é preciso manter um permanente processo educativo junto aos idosos com DCNTs, esclarecendo a condição de saúde e necessidade de tratamento. A realização de intervenções de educação em saúde pela enfermagem visa oferecer ao paciente capacidade em reconhecer a doença e entender a importância da adesão à terapia ${ }^{19}$. Os participantes demonstram saber quais são os meios para o controle das doenças, mediante orientação da equipe de enfermagem, mesmo que não façam adesão na sua plenitude do plano de cuidado. Todas as ações orientadas ao controle da DCNT funcionam como uma união de forças, aprendida e incorporada no seu dia a dia.

A partir dessas colocações, sabe-se da necessidade de um acompanhamento frequente para os idosos com HAS e DM, de modo a sensibilizá-los sobre as mudanças no estilo de vida para prevenir os agravos dessas patologias, além da importância na adesão ao plano de cuidado proposto, para que tenha uma melhor qualidade de vida. ${ }^{20}$

Dentre as repostas dos participantes foi citado também o fato da consulta de enfermagem representar a mudança que eles estavam precisando na sua vida, mudança no modo de viver, no modo de se cuidar. Notamos que o processo de educação em saúde se torna muito mais fácil, quando o enfermeiro consegue manter uma relação de empatia com o idoso durante o acompanhamento no programa Hiperdia.

Para que ocorra essa mudança na vida dos idosos, os enfermeiros trabalham com um dos princípios fundamentais do SUS, que é a integralidade. A importância de abordar a assistência de forma integrada é baseada na articulação de todos os passos na produção do cuidado e no restabelecimento da saúde. Propõe-se mapear a integralidade da assistência pelo acompanhamento da linha do cuidado, evitando-se assim a sua fragmentação.

Desta maneira, o enfermeiro atua com um plano terapêutico individualizado, no qual visa à mudança necessária para as modificações na vida do idoso do programa Hiperdia, trabalhando não apenas a doença, mas sim a pessoa, de forma que ele possa ser integrado na terapêutica, não apenas como coadjuvante e sim como protagonista.

Faz-se necessário muito empenho nestas ações, afinal uma mudança no estilo de vida de uma população é algo que se 
consegue em longo prazo, por ser difícil o acesso e a aceitação da população, em geral. É de extrema importância a atuação de uma equipe multiprofissional para a realização de programas educacionais, tendo em vista minimizar o desconhecimento da população a respeito das DCNTs.

\section{CONCLUSÃO}

A consulta de enfermagem na atenção primária ao portador de hipertensão e diabetes, através do programa Hiperdia se mostrou fundamental. Ainda necessita de aperfeiçoamentos, contudo, as ações executadas já demonstram resultados expressivamente positivos.

Os idosos do Hiperdia relataram a importância do enfermeiro durante todo o tratamento, sendo citado como um profissional que avalia, cuida, conversa, orienta, se preocupa com a alimentação, com a forma certa de se medicar e que corrige se necessário, estimulando o autocuidado para que o usuário possa ter autonomia e conhecimento quanto sua doença e tratamento.

Comprovamos através dos relatos que a contribuição do enfermeiro para a mudança no estilo de vida foi fundamental, foram inúmeros os relatos positivos quanto a melhoria no estilo de vida que notamos que estavam relacionados com mudança na alimentação, melhora na qualidade de vida, adoção de hábitos saudáveis como exercícios físicos regulares e o cuidado quanto a ingestão de medicamentos.

Dessa forma, os enfermeiros são fundamentais para o tratamento dos idosos do programa Hiperdia, visto que é um profissional capacitado para fazer as orientações e cuidados necessários, para a promoção da saúde dos portadores de HAS e DM. Seria importante uma maior valorização desse profissional, para que possam desenvolver tecnologias capazes de contribuir para a transformação do estilo de vida dos idosos.

Como trata-se de um estudo qualitativo com os idosos de uma unidade de saúde, houve a limitação sobre a real representatividade da consulta de enfermagem do município, uma vez que as condutas de enfermagem são diferenciadas de acordo com o profissional que executa o processo de enfermagem. Assim, sugere-se uma pesquisa ampla sobre a temática para maior compreensão do fenômeno, de maneira que possa melhorar assistência de enfermagem prestada aos idosos do programa Hiperdia.

Conflitos de interesses: Os autores declaram que não há conflitos de interesses

\section{REFERÊNCIAS}

1. Ministério da Saúde (Brasil), Secretaria de Atenção à Saúde. Departamento de Atenção Básica. Estratégias para o cuidado da pessoa com doença crônica: hipertensão 
arterial sistêmica [Internet]. Brasília, DF: Ministério da Saúde; 2013 [citado em 29 jan 2019]. 128p. (Cadernos de atenção básica; n. 37). Disponível em: http://bvsms.saude.gov.br/bvs/publicacoes/ estrategias_cuidado_pessoa_doenca_cronic a.pdf

2. Silveira EL, Cunha LM, Pantoja MS, Lima AVM, Cunha ANA. Prevalência e distribuição de fatores de risco cardiovascular em portadores de doença arterial coronariana no Norte do Brasil. Rev Fac Ciênc Méd Sorocaba [Internet]. 2018 [citado em 30 jan 2019]; 20(3):172-8. http://doi.org/10.23925/19844840.2018v20i3a9

3. Ministério da Saúde (Brasil), Secretaria de Atenção à Saúde, Departamento de Análise de Situação de Saúde. Plano de ações estratégicas para o enfrentamento das doenças crônicas não transmissíveis (DCNT) no Brasil 2011-2022 [Internet]. Brasília, DF: Ministério da Saúde; 2011 [citado em 30 jan 2019]. 160p. (Série B. Textos básicos de saúde). Disponível em: http://bvsms.saude.gov.br/bvs/publicacoes/ plano_acoes_enfrent_dcnt_2011.pdf 4. Dias KCCO, Lopes MELL, Zaccara AAL, Duarte MCS, Morais GSN, Vasconcelos MF. O cuidado em enfermagem direcionado para a pessoa idosa: revisão integrativa. Rev Enferm UFPE on line [Internet]. 2014 [citado em 30 jan 2019]; 5(8):1337-46. Disponível em:

https://periodicos.ufpe.br/revistas/revistaen fermagem/article/view/9818/9999. doi: 10.5205/1981-8963-v8i5a9818p13371346-2014

5. Carvalho Filha FSS, Nogueira LT, Viana LMM. Hiperdia: adesão e percepção de usuários acompanhados pela Estratégia Saúde da Família. Rev Rene 2011; 12(N Esp):930-36

6. Pereira RTA, Ferreira V. Nursing consultation in family health strategy. Rev Uniara [Internet]. 2014 Jul [citado em 30 jan 2019]; 17(1):99-111. Disponível em: http://revistarebram.com/index.php/revista uniara/article/view/10/7. doi: https://doi.org/10.25061/2527-

2675/ReBraM/2014.v17i1.10

7. Barbiani R, Nora CRD, Schaefer R.

Nursing practices in the primary health care context: a scoping review. Rev Latinoam Enferm. [Internet]. 2016 [citado em 30 jan 2019]; 24:e2721. Disponível em: http://www.scielo.br/pdf/rlae/v24/pt_01041169-rlae-24-02721.pdf

8. França DJR, Nunes JT, Fernandes MNF. As contribuições do cuidado ao idoso no programa de HIPERDIA, para a formação profissional. Rev Kairós [Internet]. 2014 [citado em 30 jan 2019]; 17(2):315-27. Disponível em:

http://revistas.pucsp.br/kairos/article/view/ 21750/16024

9. Bardin L. Análise de conteúdo. São Paulo: Edições 70; 2011. 10. Reis APA, Pimenta TR, Rossi VEC, Maia MAC, Andrade RD. Hipertensão arterial e diabetes mellitus: sistematização da assistência através da consulta de enfermagem em uma unidade escola do programa de saúde da família. Ciência et Praxis, 2014; 7(13): 55-62.

11. Reis J, Delgado S, Monteiro V. Promoção do autocuidado da pessoa com diabetes mellitus: da hospitalização ao domicílio. [Monografia]. Cabo Verde, África: Universidade do Mindelo; 2013. 12. Acioli S, Kebian LVA, Faria MGA, FerraccioliI P, Correa VAF. Práticas de cuidado: o papel do enfermeiro na atenção básica. Rev Enferm UERJ. [Internet]. 2014 [citado em 30 jan 2019]; 22(5):637-42. doi: http://dx.doi.org/10.12957/reuerj.2014.123 38

13. Waldow VR. Bases e princípios do conhecimento e da arte da enfermagem. Petrópolis: Vozes; 2008.

14. Wassenaar A, Van den Boogaard M, Van der Hooft T, Pickkers P, Schoonhoven L. Providing good and comfortable care by building a bond of trust': nurses views regarding their role in patients' perception of safety in the intensive care unit. J Clin Nurs. [Internet]. 2015[citado em 30 jan 2019]; 24(21-22):3233-44. Disponível em: 
https://onlinelibrary.wiley.com/doi/epdf/10 $.1111 /$ jocn. 12995

15. Lima MJ. O que é enfermagem. São Paulo: Brasiliense; 2017.

16. Viana TCT, Medeiros LB, Novais K M, Silva, ALG, Pipper SO, Silva MV. Qualidade de vida dos idosos cadastrados no programa hiperdia de uma unidade básica de saúde na região norte. Revista Eletrônica Acervo Saúde [Internet]. 2019; 19 Suppl:e224. doi:

https://doi.org/10.25248/reas.e224.2019 17. Lavie CJ, Arena R, Swift DL, Johannsen NM, Sui X, Lee DC, et al. Exercise and the cardiovascular system: clinical science and cardiovascular outcomes. Circ Res. [Internet]. 2015 [citado em 30 jan 2019]; 117(2):207-19.

Disponível em:

https://www.ahajournals.org/doi/pdf/10.11

61/CIRCRESAHA.117.305205. doi:

10.1161/CIRCRESAHA.117.305205

18. Radovanovic CAT, Santos LA, Carvalho MDB, Marcon SS. Hipertensão arterial e outros fatores de risco associados às doenças cardiovasculares em adultos.

Rev Latinoam Enferm. [Internet]. 2014 [citado em 30 jan 2020]; 22(4):547-53.

Disponível em:

http://www.scielo.br/pdf/rlae/v22n4/pt_01

04-1169-rlae-22-04-00547.pdf. doi: http://dx.doi.org/10.1590/0104-

1169.3345.2450

19. Bezerra ASM, Lopes JL, Barros

ALBL. Adesão de pacientes hipertensos ao tratamento medicamentoso. Rev Bras

Enferm. 2014; 67(4):550-55. doi:

http://dx.doi.org/10.1590/0034-

7167.2014670408

20. Motta MDC, Peternella FMN, Santos

AL,Teston EF, Marcon SS. Educação em saúde junto a idosos com hipertensão e diabetes: estudo descritivo. Revista

UNINGÁ Review [Internet]. 2018[citado em 30 jan 2019]; 18(2):48-53. Disponível em:

http://revista.uninga.br/index.php/uningare views/article/view/1502/1117

RECEBIDO: $16 / 05 / 2019$

APROVADO: $23 / 07 / 2019$

PUBLICADO: $12 / 2019$ 